\title{
EDITORIAL
}

\section{The Kinemage: A Tool For Scientific Communication}

Kinemages have been the hallmark of Protein Science from its very beginning. In fact, the title of the present editorial has been lifted from an article by David and Jane Richardson published in the inaugural issue of the Journal (Richardson DC and JS. 1992. Protein Sci 1:3-9.) This innovation has distinguished Protein Science from all other scientific journals. In recognition of their contribution, Jane and David Richardson were chosen the recipients of the 1995 AMGEN Award of The Protein Society. The widespread acceptance of the kinemages by authors and readers of Protein Science soon required additional expertise for their design and preparation and in 1993, Beth Cader Villafranca joined the Journal as an additional Graphics Consultant. Her outstanding dedication and contributions to the Journal in that capacity made it possible to increase the number of kinemages which accompanied each mailing in the form of a diskette. Eventually, these were replaced by special files in the electronic edition of Protein Science. In 1995, the team of kinemage specialists was augmented by the appointment of Kim Gernert and that team, aided by Steve White and Louise Falevsky at the University of California at Irvine, deserves credit for designing and producing the kinemage series over the years. This Editorial is dedicated to the recognition of their contribution. No words of thanks are adequate to express to David and Jane Richardson, Beth Villafranca, and Kim Gernert our appreciation for this outstanding series, made possible by "a simple and transparent display program in an easily readable and editable distribution format that can be used in commonly available personal computers" [quoted from their 1992 publication (vide supra)].
It was, therefore, with great reluctance and regret that the Council of The Protein Society, in consultation with the Editor-in Chief, decided to discontinue as of October 1, the professionally designed kinemages and to limit the series to author-designed images. There were several reasons for this decision beyond the need to re-direct resources to other purposes. Before reaching this decision, the Society and the Journal carried out a telephone survey of a randomly selected subset of Journal subscribers, the results of which were quite clearcut. While, on the one hand, most individuals felt that the kinemages were a valuable asset of Protein Science, very few of those polled stated that they actually used kinemages on a regular basis. Also, computers and molecular graphics software have improved tremendously during the past five years so that alternative technologies are now generally available. Kinemages are now commonly used for teaching and scientific illustrations and those interested in using them for these purposes can acquire the skills and experience that permit them to make their own. We do hope that authors of articles that are significantly aided in clarity of communication by an accompanying kinemage will design and submit their own with their manuscript.

We shall miss the advice and dedication of our graphics consultants and their collaborators and thank them once more for their outstanding service.

HANS NEURATH

Editor-in-Chief 